\title{
SPATIAL DISTRIBUTION OF QUASARS IN THE LBQS SAMPLE
}

\author{
YAOQUAN CHU and JIANHUI TAO \\ Center for Astrophysics \\ University of Science and Technology of China \\ Hefei, Anhui 230026, China
}

The information of spatial distribution of quasars would be very important in building the model of formation of AGN. Following the development of high efficiency detectors and related technique, quasar samples with less selection effect were and will gradually available. As we know that the power spectrum analysis gives a more robust determination of large scale distribution of objets. We develop a new methods to determine the power spectrum in a three dimension ball, which is more suited to the case of quasars survey. The details of our approach can be found in Tao and Chu (1993).

The basic simplified formula for a part of the ball is:

$$
P=\left\langle\frac{\left|b_{l k}^{m}-n I_{l k}^{m}\right|^{2}}{J_{m}^{l}}\right\rangle / n-1
$$

where

$$
\begin{aligned}
b_{l k}^{m} & =\sum_{i=1}^{N} \frac{1}{r_{i} \sqrt{r_{0}}} e^{i k\left(r_{i}-R\right)} Y_{l}^{m}\left(\theta_{i}, \phi_{i}\right), I_{l k}^{m}=\int W(\Omega) \frac{1}{r \sqrt{r_{0}}} e^{i k(r-R)} Y_{l}^{m}(\theta, \phi) d V \\
J_{l}^{m} & =\int_{\Delta \Omega}\left|Y_{l}^{m}\right|^{2} d \Omega
\end{aligned}
$$

We use these methods to analysis the space distribution of quasars in the sample of Large Bright Quasar Survey (LBQS), The LBQS survey cover about 800 degree on the sky which contain about 1000 quasars with redshift $0.2<z<3.3$.

We have calculate the power spectrum for all 18 fields in LBQS sample and find no strong evidence for clustering. For all fields, $P \approx 0.2$ and no any tendency to decrease at the scale of $15 \mathrm{Mpc}$ have been found. We also check the influence of the survey border, the value of $q_{0}$, and the error in determining of redshift, and find no one of these seriously effect our statistical results.

\section{References}

1. Tao J. and Chu, Y., 1993, Scientia Sinica, in press

2. Foltz C. B. et. al. AJ. 94 (1978), p. 1423; 98 (1989), p. 1959; 101 (1991), p. 1121.( LBQS)

\section{0}

T. J.-L. Courvoisier and A. Blecha: Multi-Wavelength Continuum Emission of AGN, 500.

(C) 1994 IAU. Printed in the Netherlands. 